MODELING, IDENTIFICATION AND CONTROL, 1990, vOL. 11, NO. 1, 3-11

doi:10.4173/mic.1990.1.1

\title{
Structural solution of highly redundant sensing in robotic systems
}

\author{
JENS G. BALCHEN $\dagger$ and FREDRIK DESSEN $\dagger$
}

Keywords: Robots, sensory systems, redundancy.

Reasons for and problems concerning the integration of large number of sensors in robotic systems are discussed. These include data collection, coordination and integration by means of state estimation. The need for unified approaches in handling highly redundant sensory systems is stated and solutions suggested.

\section{Introduction}

Highly redundant sensing (HRS) in robotic systems is of interest for a number of reasons. High redundancy in this context means the use of maybe ten times as many sensors as is necessary to control each degree of freedom. Some reasons for discussing HRS are:

Many less accurate sensors may cost less than a few accurate sensors.

Possibility of higher insensitivity to sensor failure.

Possibility of distributed sensing giving better control in narrow and complicated environments.

Possibility of better control of robotic systems with a high number of degrees of freedom.

Possibilities for new sensor designs.

It is important to explore new ideas with their advantages and disadvantages at an early stage of development, particularly from a theoretical point of view so that practical development of equipment as well as software, is done along the most promising lines. This paper outlines basic problems met when incorporating large number of sensors in robotic systems and a number of structural solutions are suggested which may form bases for future development.

\section{Types of sensors in HRS systems}

Practically any kind of sensor now available is a candidate for an HRS system, but it is also likely that new sensor principles may emerge as a consequence of the HRS principle. To justify the introduction of large numbers of one or more sensor types, these must each be relatively cheap. This rules out high redundancy in, for instance, resolvers and 6 d.o.f. force sensor units. In robotics today it is common to supply a system with just enough sensors to complete its single class of tasks. Furthermore, the only standard sensors on a manipulator are those for joint motion measurements. Additional sensors, such as for force sensing or collision detection

Received 5 November 1988.

$\dagger$ Division of Engineering Cybernetics, The Norwegian Institute of Technology, 7034 Trondheim, Norway.

This paper was presented at the NATO Advanced Research Workshop held at Ilciocco, Italy October 1988 and is reprinted with permission of Springer Verlag. 
are purchased separately, and usually function as separate units. Basic to HRS is not only the use of large numbers of sensors, but also multiple usage of each device. Often a sensor has a specific primary function. Due to coupling however, it can be influenced by effects other than those desired. Common approach in such cases is either to neglect the coupling or to eliminate it through filtering. A more positive, however less common approach will be to take side effects into account in order to collect as much information as possible from the complete system. In this way, a force sensor could also function as an accelerometer whereas collision detectors could be used to collect information on the environment.

An HRS system should be relatively open and contain a certain amount of general sensors for the detection of mechanical quantities such as position, velocity and acceleration as well as forces, torques and mechanical strain distribution. The sensors may either be separate or form groups of related elements. An example of the latter is a camera which may be regarded a general $n \times m$ multiple sensor since it actually receives this number of independent pieces of information. A sensor may also be as primitive as the hair of a caterpillar which when touched, produces a signal from a nerve cell. A microswitch bears some similarity to this biological device. It is believed that extensive use of miniaturized sensors based on integrated circuit technology will be characteristic in HRS. As an example, a simple accelerometer may consist of a small mass mounted on a small force sensor. A number of these devices, plus perhaps a number of distributed strain gauges, would be helpful in the control of non-rigid manipulators.

Because of the great variety and varying degree of complexity among sensing devices, it appears that an attempt to generalize their characteristics will not be very fruitful. Rather it is suggested in a later section that mathematical modeling of the actual sensing device combined with an estimation scheme is a powerful way of integrating sensors of widely different character.

\section{Data collection and fusion}

It is desirable to have a standard approach to sensor data conversion, multiplexing, transmission, intermediate storage and retrieval, see Fig. 1. Available concepts, devices and protocols make all this possible, but a remaining obstacle is price in relation to performance. With present trends in the development of VLSI chip production techniques it may be expected that each sensing device with an information capacity above a certain lower limit is equipped with a nodal device which connects it to a digital network which may be serial or parallel in nature. It is a

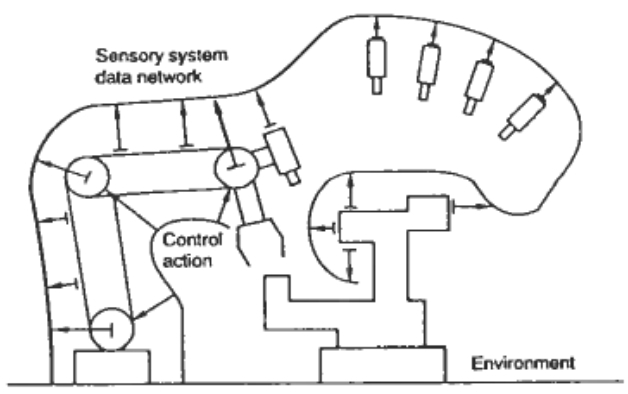

Figure 1. Collecting data on the robot and its environment. 
matter of price/performance ratio how complex the sensor should be before it justifies this approach. Sensor elements with lower information capacities may be divided into groups which serve under more general nodal devices in the same network.

One crucial question which is also related to the price/performance-ratio of the data conversion-connection-transmission concept mentioned above, is to what extent local 'intelligence' should be applied to the primary data from the sensor or a group of sensors before transmission takes place to the central processor. Obviously no general answer exists to this question because it depends on the processes involved.

The principles of sensor data fusion on a local level may well be the same as those employed on a higher level, which will be discussed in the following paragraphs, or they may involve only elementary filtering and formating. Several reasons exist for introducing data fusion, the most obvious one being data compression. As an example, this is common in image processing where a frame often may be broken into objects and sets of object features. Additional compression may be accomplished by using Kalman filter theory and only transmitting the difference between actual and predicted data. This may either be done for the complete frame or for separate objects and object features of interest. Secondly, data fusion may be used to obtain more robust data. This is often done by analog rather than digital computation, but not always. An example of the first is a measurement bridge where fusion of four elementary sensors into one output voltage is carried out. Further robustness is obtained by considering the output relative to the bridge supply voltage.

\section{Coordinate transformation}

In most robotic systems there is an extensive demand for coordinate transformation both in the interpretation of measurement data and in the execution of control actions. Many alternative coordinate systems may be used to describe the movement of a robotic system and these will have advantages and disadvantages depending on the particular task at hand. Considering the possibility of rapid shifts between working coordinate systems during the completion of a task and the fact that sensors may be fixed to any of these and several others, the need for keeping track of relationships in a structured way becomes obvious. Figure 2 indicates how these relationships may form a tree. Here each node represents a coordinate frame

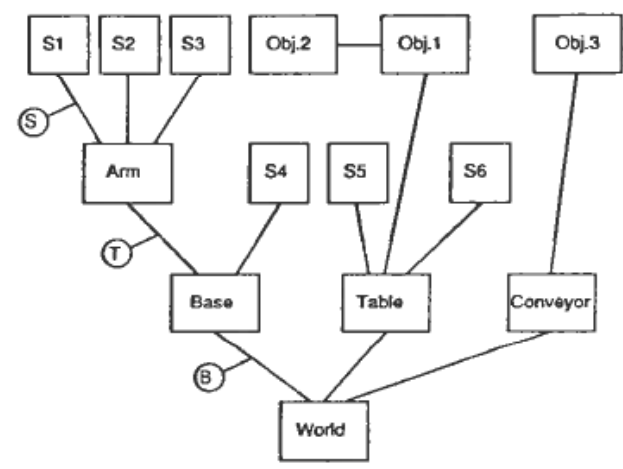

Figure 2. Representation of coordinate frames. 
and each link a kinematic relationship. The latter may be given in terms of homogeneous transformations and translational and angular velocities. These may be functions of the system state vector defined in the next section.

At present, the scope is to show how this representational form enables a structured treatment of sensor data. Assuming in Fig. 2 that sensor S1 at the moment presents the positional vector $p$, its representation in say WORLD coordinates is obtained by successive transformation of $p$ along the path between $\mathrm{S} 1$ and WORLD. In this case the result is (Paul 1981)

$$
{ }^{\text {WORLD }} p=\text { BTS } p
$$

Generally, eacin link in the tree may be represented by logical transformation machines, forming a computational pipeline. The presented structure indicates the possibility of transmitting data packages from an arbitrarily large number of different sensors through a network of self-supported relays. These machines may either be software or hardware devices. The indicated transformation machine is sufficiently general to become a standard VLSI device for robotics and computer graphics, and will in that case be available at a reasonable price.

\section{Cost optimal state estimation}

A main objective of installing sensors in robotic systems, is to facilitate the determination or estimation of the necessary number of state variables of the robotic system and its environment in order to control the system behaviour. A robotic system with 6 degrees of freedom should at least be characterized by 12 state variables in order to derive adequate control. Sometimes state variables describing the environment have to be included as well, representing stationary or moving obstacles to be avoided or workpieces with which the robotic system shall interact. A sensing system then will produce signals that are related to these state variables. Preferably these relationships should be simple, i.e. few state variables enter into the functional description. It is soon discovered however, that when modeling a sensing device, the relationships between the system state variables and the sensor output may become quite complex. Simplification of the sensing model therefore is an issue leading to the need for redundancy in order to acquire a good accuracy.

Provided that the sensor signals are means to determine the state variables, an inversion of the sensor data into state variable data is required. If the relationships between the state variables and the sensor data are linear, and there are no dynamic effects involved in the sensing, a least squares inversion algorithm may be derived as follows. If

$$
\begin{aligned}
& y: \text { sensor output vector }(\operatorname{dim} y=m) \\
& y_{0}: \text { sensor bias vector }\left(\operatorname{dim} y_{0}=m\right) \\
& x: \text { state vector }(\operatorname{dim} x=n) \\
& w: \text { uncertainty vector }(\operatorname{dim} w=m) \\
& D: \text { sensor model matrix }(m \times n)
\end{aligned}
$$

The following linear vector equation may describe the sensing process:

$$
y=y_{0}+D x+w
$$

The uncertainty vector $\boldsymbol{w}$ describes the uncertainty (noise) associated with each measurement and is often assumed to be a stochastic process with zero expected value 
and having the character of white noise, that is

$$
\begin{aligned}
E(w(t)) & =0 \\
E\left(w(t) w(t+\tau)^{\mathbf{T}}\right) & =W \delta(\tau)
\end{aligned}
$$

When $m>n$, the least squares inversion algorithm can be employed to solve (1) for the unknown vector $\boldsymbol{x}$

$$
\hat{\boldsymbol{x}}=\left(D^{\mathrm{T}} W^{-1} D\right)^{-1} D^{\mathrm{T}} W^{-1}\left(y-y_{0}\right)
$$

(4) assumes that the first term is invertible, that is

$$
\operatorname{rank}\left(D^{\mathrm{T}} W^{-1} D\right)=n
$$

Redundancy appears when $m>n$ and highly redundant sensing (HRS) appears when $m \gg n$.

A solution equivalent to that of (4) can be arrived at by assuming that the state of the total system is governed by a set of differential equations

$$
\dot{\boldsymbol{x}}=A \boldsymbol{x}+\boldsymbol{v}
$$

where $v$ is a zero mean, white noise stochastic vector

$$
\left.E(v)=0, \quad E\left(v(t) v(t+\tau)^{\mathrm{T}}\right)=V \delta(\tau)\right)
$$

A Kalman filter can be employed to estimate $\boldsymbol{x}$ (Jazwinski 1970).

The continuous Kalman filter for a linear process is given by

$$
\begin{aligned}
& \hat{x}(t)=A \hat{x}(t)+K(t)(y(t)-D \hat{x}(t)) \\
& K(t)=X(t) D^{\mathrm{T}} W^{-1} \\
& \dot{X}(t)=A X(t)+X(t) A^{\mathrm{T}}-X(t) D^{\mathrm{T}} W^{-1} D X(t)+V
\end{aligned}
$$

where

$$
X(t)=E\left[(\hat{\boldsymbol{x}}-\boldsymbol{x})(\hat{\boldsymbol{x}}-\boldsymbol{x})^{\mathrm{T}}\right]
$$

is the covariance matrix of the state vector estimate. As seen from (9), $X$ is found by solving the non-linear matrix Riccati equation. Given the values of $D$ and $W$, it is a minimum-variance estimate.

When designing an HRS system, several alternatives exist for the dimensions and the values of $D$ and $W$. The final choice will be a result of considering aspects such as price, variance and insensitivity with respect to sensor failure. Since at the moment a linear system is considered, a relatively simple optimization problem results once an appropriate objective functional is stated. This is of course a problem in itself but may be enlightened by considering the situation below.

For simplicity, the first order dynamic system

$$
\dot{x}=a x+v
$$

is assumed to be observed through a number of direct measurements

$$
y=[1,1, \ldots, 1]^{\mathrm{T}} x+w
$$

From this and (9) the equation

$$
\dot{X}=2 a X-\tilde{k} X^{2}+V
$$


is obtained where $\tilde{k}$ is the sum of all elements of the $m \times m$ matrix $W^{-1}$. Assuming further that the elements of $\boldsymbol{w}$ are uncorrelated and equal in variance, $\tilde{k}$ is related to the quality of each sensor by

$$
\tilde{k}=\frac{m}{\sigma_{w}^{2}}
$$

Under stationary conditions, by (13), $\tilde{k}$ is related to the quality of the state estimate as

$$
\tilde{k}=(2 a X+V) / X^{2}
$$

It is very reasonable that the price of a sensor will be a function $p\left(\sigma_{w}\right)$ of the accuracy which increases as the inaccuracy $\left(\sigma_{w}\right)$ approaches zero. The total price of the sensing system will become

$$
P=p\left(\sigma_{w}\right) m=\tilde{k} \sigma_{w}^{2} p\left(\sigma_{w}\right)
$$

on $\sigma_{w}$. Depending on $p($ ), the total price may or may not exhibit a minimum at a certain value of $\sigma_{w}$ (Fig. 3). Often the problem during the design phase is to obtain a specific $X$ at the lowest possible cost. By (15) this results in a specific $\tilde{K}$ which by (16) is proportional to the optimal price of the system.

From this it is obvious that in certain cases, in order to increase the accuracy of a state estimate, it will be more profitable to increase the number of sensors rather than choosing more exact devices. Whether or not this number of sensors results in

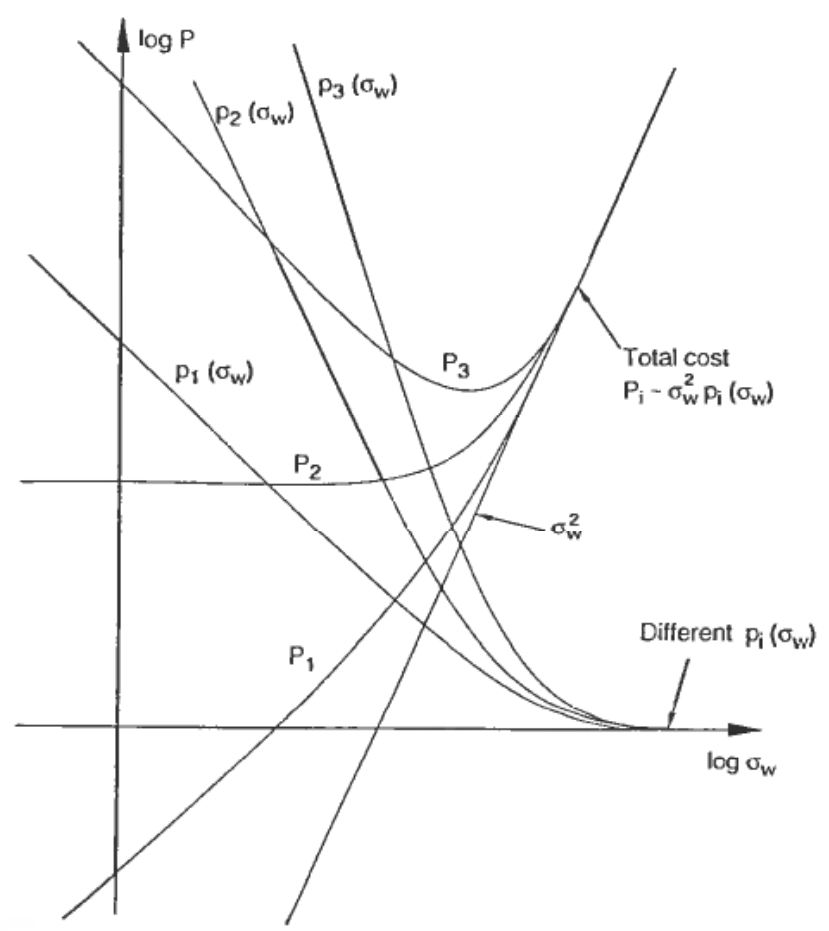

Figure 3. Sensor price functions, and their relation to total cost. 
redundancy is a question of definition. In any case, the integration of large number of possibly cheap inaccurate sensors create a special situation.

\section{Estimation problems in HRS}

Just to make it simple, the analysis above was based upon a linear dynamic system with linear sensor models. In practical robotics this is not realistic since most sensors, and the manipulator dynamics as well, will be described by non-linear relationships.

In formulating a non-linear model for a robot and its environment, it may be advantageous to divide the total state of movement into for instance four substrates $x_{1}, x_{2}, x_{3}$ and $x_{4}$ where $x_{1}$ and $x_{3}$ represent the positional states of the robot and the environment respectively whereas $x_{2}$ and $x_{4}$ are the equivalent velocity vectors. Therefore a mathematical model of the total system will have the form

$$
\begin{aligned}
& \dot{x}_{1}=x_{2} \\
& \dot{x}_{2}=f_{2}\left(x_{1}, x_{2}, u, v_{1}\right) \\
& \dot{x}_{3}=x_{4} \\
& \dot{x}_{4}=v_{2}
\end{aligned}
$$

In (19) it is assumed that the acceleration vector is a non-linear function $\left(f_{2}(\cdot)\right)$ of both position and velocity states, the control vector $(u)$ and a zero mean white noise disturbance vector $\left(v_{1}\right)$. In (21) it is assumed that the environment acceleration vector is a zero mean white noise vector $\left(v_{2}\right)$.

The model of the sensors is divided into two parts, one concerned with the sensing of the robot motion itself and the other describing the sensing of the environment. Thus we have

$$
\begin{aligned}
& y_{1}=g_{1}\left(x_{1}, x_{2}\right)+w_{1} \\
& y_{2}=g_{2}\left(x_{1}, x_{2}, x_{3}, x_{4}\right)+w_{2}
\end{aligned}
$$

Here $\boldsymbol{w}_{1}$ and $\boldsymbol{w}_{2}$ represent uncertainties with respect to the different sensing functions and are regarded as zero mean white noise vectors. The functions $g_{1}(\cdot)$ and $g_{2}(\cdot)$ may be non-linear and even discontinuous, as is the case with proximity sensors, contact force sensors etc. It will appear that the derivatives of $g_{1}$ and $g_{2}$ are of importance, which implies that the functions should be relatively smooth. However, the presence of large numbers of simple sensors is characteristic for HRS. Because of this, it must be possible to handle elements such as switches and verylow-resolution digital sensors with or without hystereses. Thus to design the system, some knowledge on non-linear control theory will be required.

Figure 4 shows a block diagram of a total system state estimator utilizing the sensor outputs $y_{1}$ and $y_{2}$ and estimating $x_{1}, x_{2}, x_{3}$ and $x_{4}$. The feedback strategy can be determined on the basis of Extended Kalman Filter theory, or by other means. The estimator can also be used for sensor failure detection (Willsky 1976; Mehra and Peschon 1971).

In the actual implementation of the functions of Fig. 4 and the feedback strategy, it will be possible to make a number of simplifications to reduce the computational complexity. One extreme type of simplification would be to regard the covariance matrix $X$ in (9) as a constant. Computational simplifications may be considered in 


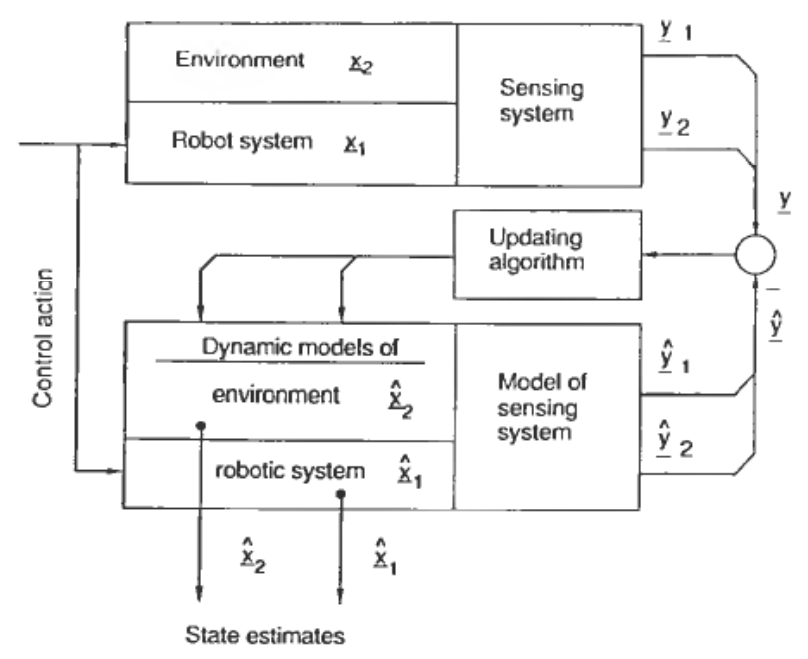

Figure 4. Extended Kalman filter.

each case, and are highly recommended. The possibility of distributed estimation should especially be considered. It must be remembered that the large number of sensors involved ensures that the quality of one single update is not critical. In terms of (16), a close correlation exists between the price of the measurement update and the computational complexity, counting hardware and software development costs. The sensor price function $p_{i}\left(\sigma_{i}\right)$ may now be found for specific $\sigma_{i}$ by considering the corresponding mechanical and computational complexity, and the optimal choice found by considering (10) and Fig. 3.

The large amount of sensors assumed makes the use of multiplexing inevitable. This creates a situation where data are highly skewed. Depending on the use of each sensor, their sampling rates may vary as well. These events may appear at fixed rates, or they may be stochastic. The latter may for instance be the case for switches and very-low-resolution digital elements, if they are set to report whenever a change in the output occurs. Taking into account the possibility of now-and-then appearing data, a structure which may be called an event-driven state estimator will result. A special case, for the estimation of static geometrical data is presented in Moravec (1987).

\section{Conclusion}

Means have been discussed for employing large numbers of sensors in robotic systems. Throughout, the discussion has concentrated on the estimation of the state in the systems' equations of motion. Similar considerations can be made concerning the estimation of its parameters and the determination of its structure. Special cases along this lane include object recognition and model building. In any case, it is obvious that the incorporation of large numbers of sensors can be beneficial. A question yet unanswered concerns redundancy.

Taking the point of view that redundancy is a task related concept, it is obvious that if the robot task can be fulfilled using a certain set of sensors, additional sensors will be redundant. The degree of sensory redundancy may be determined in terms of observability and identifiability in control theory. These reflect the existence of 
inverse relationships between the sensory information and the states, parameters or features of the system. Thus, a highly redundant sensory system can be able to function even if a large number of its sensors malfunction. The degree of taskspecific redundancy can be a matter of reliability, versatility and economical considerations.

\section{REFERENCES}

Jazwinsky, A. H., (1970), Stochastic Processes and Filtering Theory, Academic Press, New York.

MeHra, R. K., and Peschon, J., (1971), An innovations approach to fault detection and diagnosis in dynamical systems. Automatica, 7, 637-640.

MoRAVEC, H. P., (1989), Sensor fusion in certainty grids for mobile robots. Sensor Devices and Systems for Robots. NATO ASI Series, Springer Verlag.

PaUL, R. P., (1981), Robot Manipulators (MIT Press, Cambridge, MA).

WiLlsKY, A. S., (1976), A survey of design methods for failure detection in dynamic systems. Automatica, 12, 601-611. 